\title{
Journal of Neurology
}

\section{A multicenter study on the diagnostic significance of a single cerebrospinal fluid lgG band \\ --Manuscript Draft--}

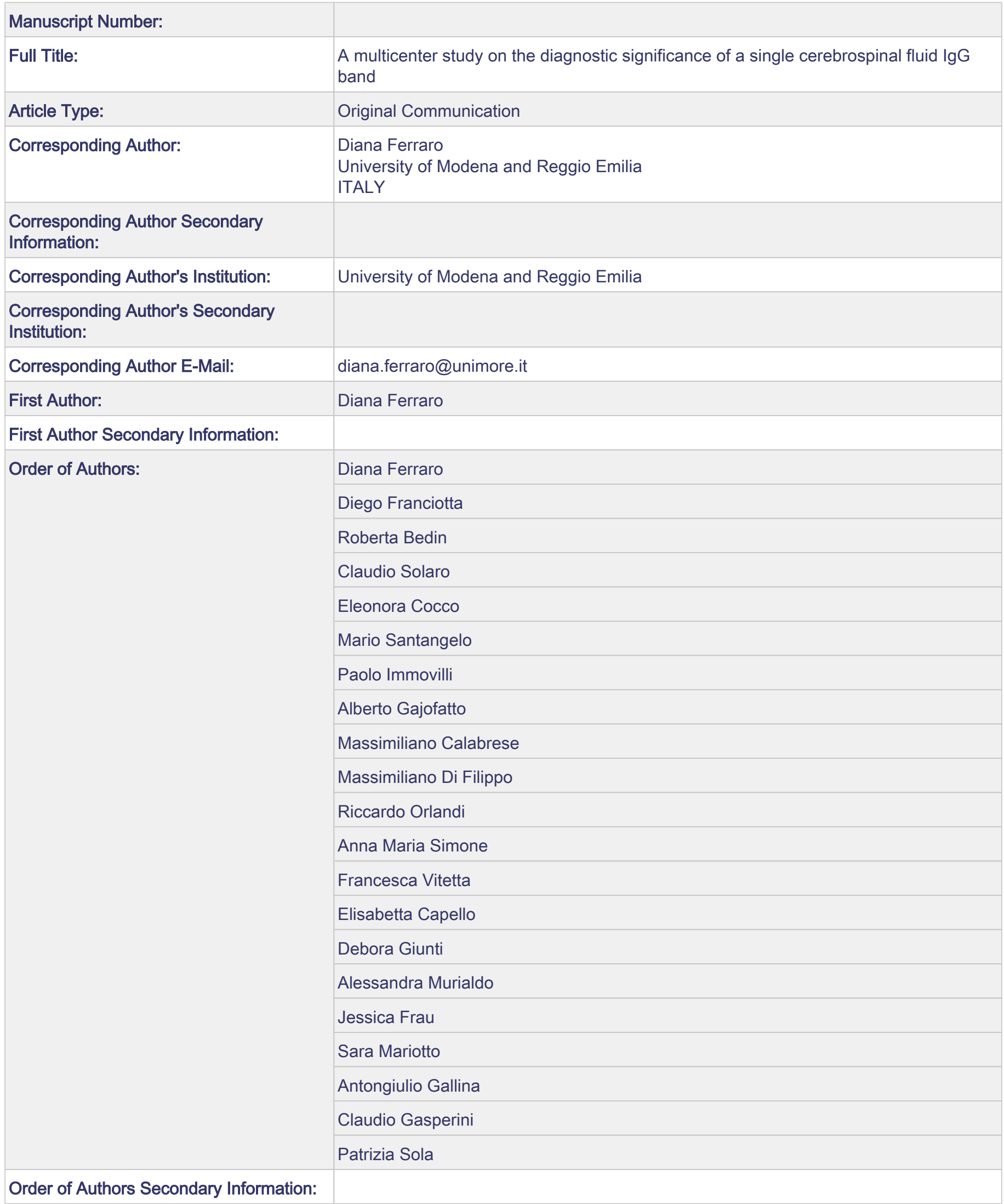


Funding Information:

Abstract:

Suggested Reviewers:

\section{Author Comments:}

The analysis of cerebrospinal fluid (CSF) and serum with isolectric focusing can be of aid in the differential diagnosis of central nervous system (CNS) disorders. However, the diagnostic meaning of a single CSF-restricted IgG band is still uncertain.

Aim of this multicenter study was to establish the frequency and disease associations of this finding.

Of 9422 CSF reports, 151 (1.6\%) showed a single CSF-restricted IgG band. Of the 129 patients with a definite diagnosis, $58.2 \%$ had CNS inflammatory-demyelinating diseases (the most frequent being Multiple Sclerosis: $21.7 \%$ ), 6.2\% tumours, $5.4 \%$ inflammatory peripheral nervous system diseases, and $30.2 \%$ miscellaneous diseases. At repeat CSF analysis, 3 out of 10 patients had developed an oligoclonal band pattern.

Our results indicate that single CSF IgG bands tend to associate with diseases characterized by an intrathecal humoral immune response. This abnormality should alert clinicians of possible inflammatory disorders of the CNS and should, therefore, be reported.

Francesco Lolli

francesco.lolli@unifi.it

Author of previous article on the same topic

Gerard Davies

gerard.davies@nhs.net

Author of only other study on the follow-up of patients with a single CSf IgG band

Hayrettin Tumani

hayrettin.tumani@uni-ulm.de

Expertise in Neuroimmunology

Olaf Stuve

olaf.stuve@utsouthwestern.edu

Expertise in Neuroimmunology

\section{Dear Sir}

we would like to submit our manuscript entitled "A multicenter study on the diagnostic significance of a single cerebrospinal fluid IgG band" for consideration by Journal of Neurology as an original article.

The detection of oligoclonal IgG bands (OCB) in paired serum and cerebrospinal fluid (CSF) samples is a valid tool in the differential diagnosis of central nervous system (CNS) disorders. Very few studies, however, have addressed the diagnostic significance of a single cerebrospinal fluid IgG band at isoelectric focusing (IEF), which falls into a grey area between 'no bands' (normality) and 'two bands' (pathological production of oligoclonal lgG within the CNS), and may even not be mentioned in the CSF analysis report.

In a multicenter study we were able to evaluate the frequency and disease associations of this finding, which was present in $1.6 \%$ of 9422 CSF analyses (151 cases). The majority of the associated diseases had an inflammatory component of the CNS (e.g. multiple sclerosis and demyelinating, autoimmune or paraneoplastic CNS diseases) and an intrathecal humoral response is, thus, deemed plausible in these cases.

We therefore strongly recommend reporting this IEF abnormality, since it can be of aid to clinicians during the diagnostic process and it should alert them of the possible presence of inflammatory CNS diseases.

We hope that you will find our work to be of interest to Journal of Neurology, in light also of the previously published article on the same topic [Franciotta D, Bergamaschi $\mathrm{R}$, Amato MP, et al (2005) Clinical correlations of CSF single lgG bands. J Neurol 252:1274-1275. doi: 10.1007/s00415-005-0820-1]

Yours sincerely,

Diana Ferraro 


\section{A multicenter study on the diagnostic significance of a}

\section{single cerebrospinal fluid IgG band}

Diana Ferraro $^{\mathrm{a}}$, Diego Franciotta ${ }^{\mathrm{b}}$, Roberta Bedin $^{\mathrm{a}}$, Claudio Solaro $^{\mathrm{c}},{\text { Eleonora } \text { Cocco }^{\mathrm{d}} \text {, Mario Santangelo }}^{\mathrm{e}}$, Paolo Immovillif $^{\mathrm{f}}$, Alberto Gajofatto ${ }^{\mathrm{g}}$, Massimiliano Calabrese ${ }^{\mathrm{g}}$, Massimiliano Di Filippo ${ }^{\mathrm{h}}$, Riccardo Orlandi ${ }^{\mathrm{g}}$, Anna Maria Simone $^{\mathrm{a}}$, Francesca Vitetta $^{\mathrm{a}}$, Elisabetta Capello ${ }^{\mathrm{i}}$, Debora Giunti $^{\mathrm{i}}$, Alessandra Murialdo $^{\mathrm{j}}, \mathrm{Jessica}$ Frau $^{\mathrm{d}}$, Sara $_{\text {Mariotto }}^{\mathrm{g}}$, Antongiulio Gallina $^{\mathrm{h}}$, Claudio Gasperinik ${ }^{\mathrm{k}}$, Patrizia Sola ${ }^{\mathrm{a}}$ and on behalf of the RIREMS group (Rising Italian Researchers in Multiple Sclerosis)

${ }^{a}$ Neurology Unit, Department of Neurosciences, Nuovo Ospedale Civile S. Agostino Estense, University of Modena and Reggio Emilia, Via P. Giardini, 1355, Modena, Italy

${ }^{\mathrm{b}}$ Laboratory of Neuroimmunology, IRCCS, National Neurological Insitute C. Mondino Foundation, Via Mondino 2, Pavia, Italy

${ }^{\mathrm{c}}$ Neurology Unit, Department of Head and Neck, ASL3 genovese, L.go Rosso, 2, Genova, Italy

${ }^{\mathrm{d}}$ Department of Medical Sciences and Public Healths, Cittadella di Monserrato,University of Cagliari, Cagliari, Italy

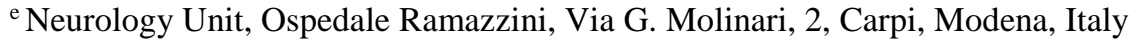

${ }_{\mathrm{f}}^{\mathrm{f}}$ Neurology Unit, Department of Specialistic Medicine, G. da Saliceto Hospital, Via T. Giuseppe, 49, Piacenza, Italy

g Department of Neuroscience, Biomedicine and Movement, University Hospital of Verona, P.le Scuro 10, Verona, Italy

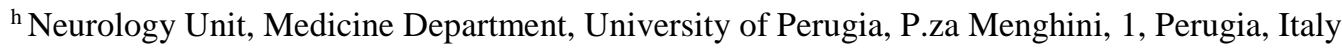

${ }^{\text {i }}$ Department of Neuroscience, Rehabilitation, Ophthalmology, Genetics, Maternal and Child Health,

University of Genova and IRCCS AOU San Martino-IST, Largo R. Benzi, Genoa, Italy

${ }^{\mathrm{j}}$ Unit of Neurology, IRCCS San Martino University Hospital IST, Largo R. Benzi, Genoa, Italy.

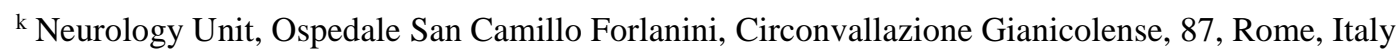

Corresponding author:

Diana Ferraro

Institution: $\quad$ Neurology Unit, Department of Biomedical, Metabolic and Neurosciences, University of Modena and Reggio Emilia, Modena, Italy 
Address: $\quad$ Nuovo Ospedale Civile Sant'Agostino Estense

1

2

3

4

5

6

7

8

9

10

11

12

13

14

15

16

17

18

19

20

21

22

23

24

25

26

27

28

29

30

31

32

33

34

35

36

37

38

39

40

41

42

43

44

45

46

47

48

49

50

51

52

53

54

55

56

57

58

59

60

61

62

63

64

65

Via Pietro Giardini, 1355

41126 Modena, Italy

Tel: $\quad+\quad+390593961722$

Fax: $\quad+390593961323$

E-mail: $\quad$ diana.ferraro@unimore.it

ORCID ID: $\quad 0000000348183806$

Key-words: cerebrospinal fluid; isoelectric focusing; single IgG band; Multiple Sclerosis 


\section{Introduction}

The detection of oligoclonal IgG bands (OCB) in paired serum and cerebrospinal fluid (CSF) samples is still considered a valid diagnostic tool in the differential diagnosis of inflammatory diseases of the central nervous system (CNS), notwithstanding the OCB 'downgrading' by the latest revision of the diagnostic criteria for multiple sclerosis (MS) (reviewed in [1]). Two important consensus papers defined the most common OCB patterns that help clinical pathologists and neurologists to interpret the results of serum and CSF analysis with the recommended isoelectric focusing (IEF) techniques [2, 3]. However, additional IEF patterns, which have not been addressed by the leading consensus papers [2, 3], can be detected in routine diagnostics [1]. In particular, the finding of a single IgG band in the CSF falls into a grey area between 'no bands' (normality) and 'two bands' (pathological production of oligoclonal IgG within the CNS). A few studies explored this grey area, and found that single CSF IgG bands associated with various diseases including CNS infections, lymphomas, demyelinating/autoimmune/paraneoplastic diseases, peripheral nervous system (PNS) diseases, and, above all, MS, with a highly variable frequency (from 0 to $44 \%$ ) [4-10]. Notably, these studies are not fully comparable, due to the use of agarose electrophoresis, on which a single band corresponds to an underlying single plasma cell clone, or of IEF, on which a monoclonal gammopathy yields 2-10 bands because of the technique's high resolving power that allows the detection of IgG microheterogeneity [1-3]. In addition to this methodological heterogeneity, since only a small number of case series have been studied so far, the frequency and diagnostic meaning of single CSF IgG bands is still an open question [1]. In our multicenter retrospective study on single CSF IgG bands we aimed to assess: a) their frequency and disease associations, b) possible differences in the demographic and other CSF features between the expectedly highly represented cohort of MS patients and patients with other diseases, and c) the evolution over time of CSF IEF patterns in a subgroup of patients who underwent follow-up spinal taps.

\section{Patients and Methods}

\subsection{Patients}

Seven Italian neurological centres (Modena, Genoa, Cagliari, Carpi, Piacenza, Verona and Perugia) participated in this retrospective study. Patients showing a single CSF IgG band associated with identical bands in CSF and serum ('mirror pattern') (Figure, a), or alone (Figure, b) between January 2005 and June 2016 were selected from the centres' databases. A single CSF IgG band within a mirror pattern was considered as if it was emerging from a polyclonal background, given that a mirror pattern is the product of a purely systemic oligoclonal B-cell activation, without 
intrathecal IgG synthesis [2, 3]. All the CSF analyses were carried out for diagnostic purposes. The study was approved

\subsection{Demographic and CSF features}

The following variables were collected: sex, age, date and rationale of CSF analysis (symptoms, diagnosis or suspected diagnosis), final confirmed diagnosis (if available), date of final confirmed diagnosis. CSF features included: CSF cell number and type, concentration of CSF total proteins, CSF/serum glucose ratio, IgG Index, CSF/serum albumin ratio, presence or absence of a 'mirror pattern' [2, 3].

\subsection{CSF data sources and methods}

Over $92 \%$ of the tested serum and CSF samples were analyzed in the three large laboratories of Modena (55\%), Genoa (22\%), and Cagliari (15\%), with home-made agarose gel-based IEF techniques (Agarose IEF, Pharmalyte $\mathrm{pH}$ 3-10 and Pharmalyte pH 8-10.5 GE Healthcare), and a very similar protocol. Briefly, electrode strips were soaked with 0.05 or $1 \mathrm{M}$ sodium hydroxide (catholyte), and 0.05 or $1 \mathrm{M}$ sulphuric acid (anolyte). After focusing, the proteins were transferred to polyvinylidene difluoride (Modena), or nitrocellulose (Genoa and Cagliari) membranes, which were incubated with polyclonal rabbit anti-human IgG (Modena: Dako) or goat anti-human IgG (Genoa: DiaSorin; Cagliari: Sigma-Pierce), and then with alkaline phosphatase- (Modena) or peroxidase- (Genoa and Cagliari) conjugated polyclonal swine antirabbit (Modena: Dako) or rabbit anti-goat (Genoa and Cagliari: Dako) IgG. Membranes were finally stained with 5Bromo-4-chloro-3-indolyl-phosphate and Nitro blue tetrazolium (Modena: Sigma-Aldrich), or with 3-Amino-9ethylcarbazole (Genoa and Cagliari: Sigma-Aldrich). Focusing was performed on a Multiphor II apparatus (GE Healthcare) in all centres. All the other samples were analyzed in the laboratories of Verona (which used IsoGel Agarose IEF pH 3-10 Lonza, 1M sodium hydroxide and 0.5M carboxylic acid, polyclonal rabbit anti-human IgG-Dako, biotinylated anti-rabbit IgG-Vectastain ABC kit and 3-Amino-9-ethylcarbazole) and Perugia (which used Hydragel 9 CSF isofocusing kit-Sebia).

Since 2000 the laboratories have been participating in external quality control schemes, annually organized by the Italian Association of Neuroimmunology (AINI). These schemes show that the analytical quality of OCB detection and the between-center agreement on the results of the external quality controls have substantially improved over the years (D. Fr., personal communication). As a proof of principle, the Laboratory of Neuroimmunology of Pavia, which is part of the AINI network, received 15 pairs of serum and CSF samples, with or without a single CSF IgG band, from the center of Modena, and blindly tested them, with a resulting strength of agreement that is considered to be 'fair' $(\mathrm{k}=0.25)$. 
This result is in line, if not better than expected, with the fact that the IEF technique has low inter-laboratory reproducibility, mainly due to the non-linearity of the $\mathrm{pH}$ gradient, and to the so called 'cathodic drift'[1] and that 'critical' CSF samples, e.g., those with single CSF IgG bands, are at a high risk of misinterpretation (reviewed in [1]).

As for the IEF track interpretation, the centers of Genoa and Modena exchanged and blindly assessed pictures of paired CSF and serum samples of 112 patients. A single $\mathrm{IgG}$ band was concordantly identified in $91 \%$ of cases (kappa of agreement, 0.82). Furthermore, the center of Modena re-tested 15 CSF and serum samples showing a single CSF IgG band, with completely matching results.

\subsection{Statistical methods}

Absolute frequencies and percentages were calculated for categorical variables and the mean ( \pm standard deviation) and/or the median ( \pm interquartile range) for continuous variables. Comparisons between groups were made using the Kruskal-Wallis test for continuous variables and Fisher's exact test for categorical variables, followed by post-hoc tests.

\section{Results}

\subsection{Diagnoses}

Out of a total of 9422 CSF analysis reports, 151 showed a single CSF-restricted IgG band (1.6\%). A definite diagnosis was available in 129/151 patients (Table 1). MS was the single disease with the highest frequency of single IgG band detection. Cumulatively, MS and CNS inflammatory-demyelinating diseases (clinically isolated syndrome, radiologically isolated syndrome and neuromyelitis optica spectrum disorder) accounted for $46.5 \%$ of all the cases (Table 1).

\subsection{Intra- and between-group comparisons}

Twenty-eight of the patients with a single CSF IgG band were diagnosed with MS, 32 with CNS inflammatorydemyelinating diseases and 69 with miscellaneous diseases. Mean age in patients with MS and in patients with CNS inflammatory-demyelinating diseases was significantly lower than in patients with all the other diseases. Patients with MS or with CNS inflammatory-demyelinating diseases had a lower frequency of single CSF IgG bands associated with a mirror pattern in comparison with patients with miscellaneous diseases (Table 2)..

\subsection{Follow-up findings}

Ten patients underwent a second spinal tap after a mean interval of 458 days (range: 22-1482) from the first one. Three patients (one with MS, one with progressive multifocal leukoencephalopathy and one with cerebral lymphoma) 
developed an oligoclonal pattern, while four patients (one with a compressive myelopathy, one with neurosarcoidosis, one with motor neuron disease and one with anti- N-methyl D-aspartate receptor antibody encephalitis - following treatment and remission) lost the single CSF IgG band, which remained unchanged in three patients with myelitis, viral encephalitis or neuro- systemic lupus erythematosus.

\section{Discussion}

Our main finding indicates that single CSF IgG bands preferentially associate with inflammatory diseases of the CNS. Indeed, nearly one half of the cases were found in patients with MS or inflammatory-demyelinating CNS diseases in, and another $12 \%$ in patients with CNS infections or autoimmune/paraneoplastic neurological diseases. A clear-cut and mature CNS-restricted oligoclonal B-cell response is expected in all these disorders (reviewed in [1]). It is thus likely that single CSF IgG bands might be the 'tip of the iceberg' of oligoclonal responses that are either mounting, or weak in nature, if lumbar puncture is performed near to the onset of the disorder. The abnormality could, therefore, indicate a 'possible' intrathecal IgG synthesis [6].

As for the mean younger age found in patients with MS or CNS inflammatory-demyelinating diseases versus all other patients, this finding likely depends on the typically younger age of MS onset. Analogously, the association between single CSF IgG bands and a mirror pattern was more frequent in non-MS pathologies, probably because in the latter conditions, including infectious disorders, a mirror pattern, which reflects a systemic B-cell activation, can be relatively common [1].

Table 3 summarizes the main findings of other studies exploring the diagnostic significance of a single IgG band. Cumulatively, these findings confirm our data on the preferential association between single CSF IgG bands and CNS inflammatory disorders. The plausibility of such an association is reinforced by the fact that 3 of 10 of our patients with repeated lumbar punctures developed OCB (one case of conversion from Clinically Isolated Syndrome to MS), which is in line with the data of the only other follow-up study on the topic [5]. The study showed that $33 \%$ of the patients with a single CSF IgG band later manifested CSF OCB (two thirds of them had inflammatory demyelinating diseases), whereas in approximately $50 \%$ of them the pattern remained unchanged [5].

The transition from single CSF IgG band patterns to full-blown OCB patterns in MS is not surprising, as many studies have shown intra-patient variations of OCB patterns in this disease over time [11-13], with a high number of intrathecal B-cell clones in patients with the longest disease duration [12] .

In very rare cases, single CSF bands can be expected in cerebral lymphomas $[5,8,14]$. In the present series too, there is a patient with a cerebral marginal zone lymphoma. Four additional patients had hematopoietic malignancies 
(lymphomas in 3 and chronic lymphocytic leukemia in one patient). Although reported in one case [15], single CSF bands in such systemic disorders seem to be incidental rather than disease-related.

On the other hand, the present and the previous studies $[4,5,7]$ show that in about one third $(17-44 \%)$ of patients with single CSF IgG bands, the associated diseases are either vascular or degenerative in nature, or they affect the PNS. An intrathecal IgG production is, therefore, unexpected.

In these cases, it is likely that finding a single CSF IgG band might derive from misinterpretations of the IEF tracks in a few cases at least, due to the artifactual banding that is typical of IEF, and as suggested by the results of the AINI external quality control schemes [1]. In the IEF-OCB field, some degree of inter-laboratory variability is more than expected (but no study has ever addressed this issue), as IEF is a technique that is inherently quite impossible to standardize. This shortcoming is an objective and insoluble limit of any multicenter study on the topic, including the present one. Both the inclusion of single CSF IgG band false positive cases, and the exclusion of false negative ones can thus have affected the study findings. However, the fact that the results did not differ substantially from those of the previously published studies [4-10], suggests that the rate of incorrect IEF track interpretation might be acceptable. Moreover, the three laboratories that examined more than $90 \%$ of paired serum and CSF samples participate in the AINI external quality control schemes, and some procedures aimed at boosting the reliability of the study have been performed.

As for all the other diseases, $5 \%$ of single CSF IgG bands were detected in patients with inflammatory PNS diseases, in line, again, with the other studies on the topic (Table 3). In this case, it is difficult to ascribe the abnormality to a weak or emerging intrathecal IgG production, as in the case of MS. Indeed, data that favour the hypothesis of a CNSrestricted intrathecal B-cell activation to intrathecal portions of peripheral nerves [5], or to PNS-CNS shared autoantigens [16] have not yet been substantiated by the evidence of full-blown CSF OCB. The very high frequency of a CSF-restricted monoclonal IgG band reported by Dalakas and Colleagues in 14/15 patients with chronic relapsing polyneuropathy [17] has never been confirmed.

CSF OCB have been described with very low frequencies in neurovascular $[10,18,19]$ and in neurodegenerative $[10$, 20] diseases, but, as in the case of patients with headache or psychiatric disorders,-single CSF IgG bands are more likely traces of previous contacts between infectious agents and the immune system (immunologic memory) than abnormalities related to ongoing intrathecal B-cell activation.

\section{Conclusion}

Our data show that the single most frequent diagnosis in patients with a single CSF-restricted IgG band is MS, followed by diagnoses of inflammatory disorders of the CNS. Overall, these findings indicate that single CSF IgG bands tend to 
associate with diseases characterized by an involvement of intrathecal humoral immune responses, and strongly support the notion that this abnormality should alert clinical pathologists and neurologists of a possible presence of inflammatory disorders of the CNS. Given such an important diagnostic weight, although rare, single CSF IgG bands should always be reported, and in uncertain clinical cases, the lumbar puncture should be repeated after 6-12 months.

\section{Figure Legend}

Figure Examples of isoelectric focusing patterns characterized by a single CSF IgG band associated with identical bands in CSF and serum ('mirror pattern') (a), or alone (b)

CSF: cerebrospinal fluid; S: serum; $\oplus$ : anode; $\ominus$ : cathode

\section{Declaration of interest}

The authors declare that they have no conflict of interest

\section{Funding}

This research did not receive any specific grant from funding agencies in the public, commercial, or not-for-profit sectors.

\section{REFERENCES}

1. Gastaldi M, Zardini E, Franciotta D (2017) An update on the use of cerebrospinal fluid analysis as a diagnostic tool in multiple sclerosis. Expert Rev Mol Diagn 17:31-46. doi: 10.1080/14737159.2017.1262260

2. Freedman MS, Thompson EJ, Deisenhammer F, et al (2005) Recommended standard of cerebrospinal fluid analysis in the diagnosis of multiple sclerosis: a consensus statement. Arch Neurol 62:865-70. doi: 10.1001/archneur.62.6.865

3. Andersson M, Alvarez-Cermeño J, Bernardi G, et al (1994) Cerebrospinal fluid in the diagnosis of multiple sclerosis: a consensus report. J Neurol Neurosurg Psychiatry 57:897-902.

4. Ben-Hur T, Abramsky O, River Y (1996) The clinical significance of a single abnormal immunoglobulin band in cerebrospinal fluid electrophoresis. J Neurol Sci 136:159-61.

5. Davies G, Keir G, Thompson EJ, Giovannoni G (2003) The clinical significance of an intrathecal monoclonal immunoglobulin band: a follow-up study. Neurology 60:1163-1166. doi: 10.1212/WNL.62.7.1237 
6. Franciotta D, Zardini E, Lolli F, et al (2004) The clinical significance of an intrathecal monoclonal immunoglobulin band: A follow-up study. Neurology 62: 675-676.

7. Franciotta D, Bergamaschi R, Amato MP, et al (2005) Clinical correlations of CSF single IgG bands. J Neurol 252:1274-1275. doi: 10.1007/s00415-005-0820-1

8. McCombe PA, Brown NN, Barr AE, Parkin L (1991) Monoclonal immunoglobulin bands in the cerebrospinal fluid. Aust N Z J Med 21:227-9.

9. Bass BH, Armstrong H, Weinshenker B, et al (1988) Interpretation of single band patterns in CSF protein electrophoresis. Can J Neurol Sci 15:20-2.

10. Link H, Kostulas V (1983) Utility of isoelectric focusing of cerebrospinal fluid and serum on agarose evaluated for neurological patients. Clin Chem 29:810-5.

11. Axelsson M, Mattsson N, Malmeström C, et al (2013) The influence of disease duration, clinical course, and immunosuppressive therapy on the synthesis of intrathecal oligoclonal IgG bands in multiple sclerosis. J Neuroimmunol 264:100-5. doi: 10.1016/j.jneuroim.2013.09.003

12. Mattson DH, Roos RP, Arnason BG (1981) Comparison of agar gel electrophoresis and isoelectric focusing in multiple sclerosis and subacute sclerosing panencephalitis. Ann Neurol 9:34-41. doi: 10.1002/ana.410090107

13. Thompson EJ, Kaufmann P, Rudge P (1983) Sequential changes in oligoclonal patterns during the course of multiple sclerosis. J Neurol Neurosurg Psychiatry 46:547-50.

14. Trip SA, Wroe SJ, Davies G, Giovannoni G (2003) Primary CNS mantle cell lymphoma associated with an isolated CSF monoclonal IgG band. Eur Neurol 49:187-8. doi: 69080

15. Ruiz P, Moezzi M, Chamizo W, et al (1992) Central nervous system expression of a monoclonal paraprotein in a chronic lymphocytic leukemia patient. Acta Haematol 88:37-40.

16. Kamm C, Zettl UK (2012) Autoimmune disorders affecting both the central and peripheral nervous system. Autoimmun Rev 11:196-202. doi: 10.1016/j.autrev.2011.05.012

17. Dalakas MC, Houff SA, Engel WK, et al (1980) CSF “monoclonal” bands in chronic relapsing polyneuropathy. Neurology 30:864-7.

18. Roström B, Link B (1981) Oligoclonal immunoglobulins in cerebrospinal fluid in acute cerebrovascular disease. Neurology 31:590-6.

19. Miller JR, Burke AM, Bever CT (1983) Occurrence of oligoclonal bands in multiple sclerosis and other CNS 
diseases. Ann Neurol 13:53-8. doi: 10.1002/ana.410130112

1

20. Janssen JC, Godbolt AK, Ioannidis P, et al (2004) The prevalence of oligoclonal bands in the CSF of patients with primary neurodegenerative dementia. J Neurol 251:184-8. doi: 10.1007/s00415-004-0296-4 
$\mathrm{pH}$

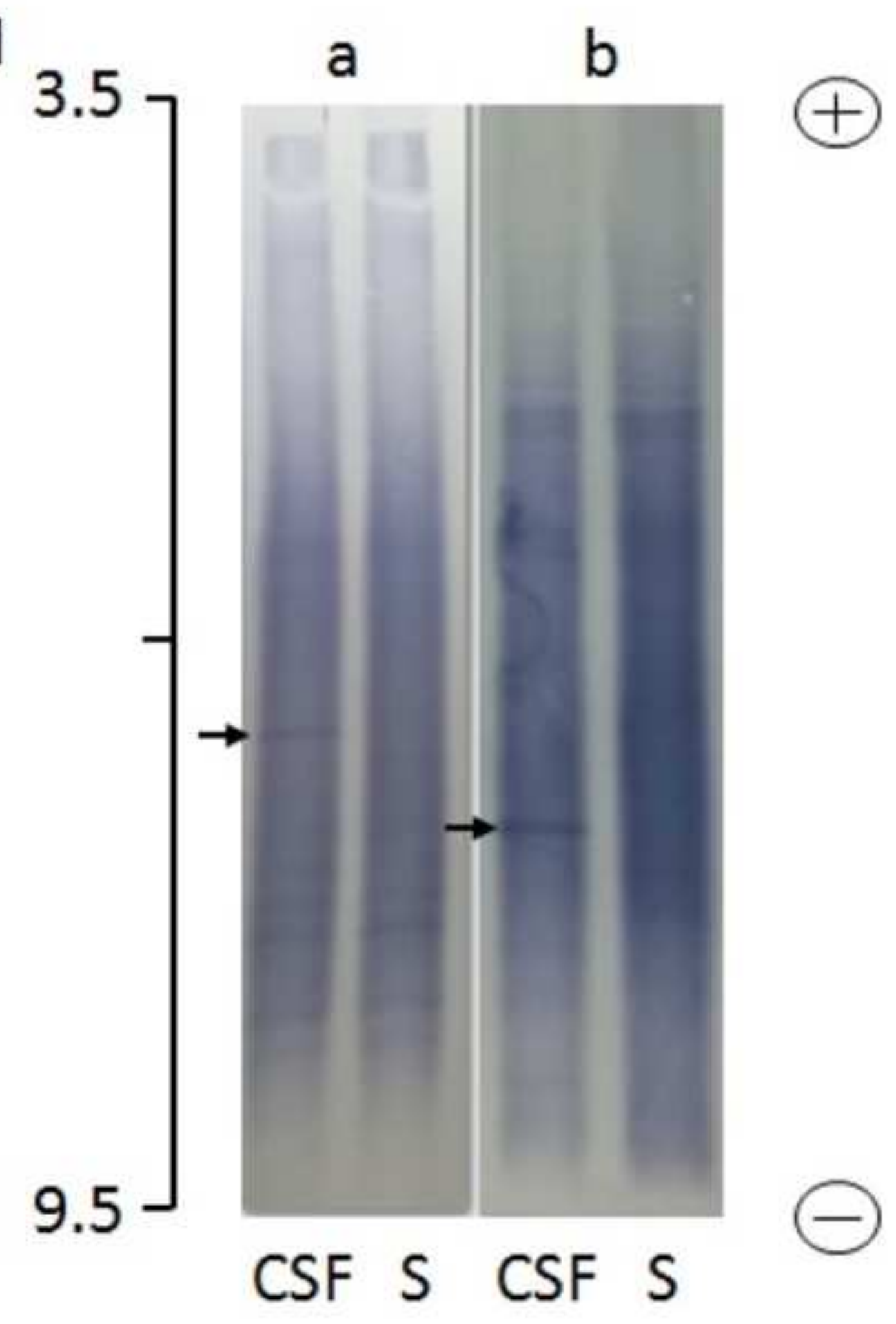
PH.

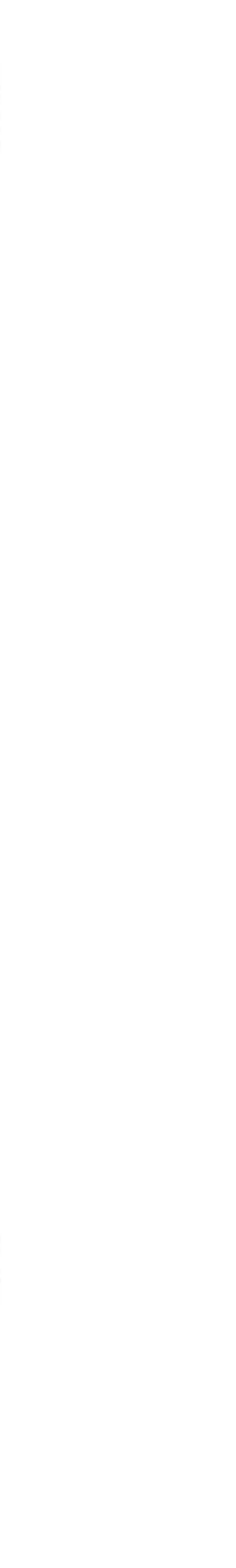

\section{.}

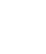

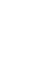


Table 1. Diagnoses associated with a single CSF IgG band in our cohort of 129 patients

\begin{tabular}{|l|l|}
\hline Diagnosis & $\begin{array}{l}\text { Number of } \\
\text { patients (\%) }\end{array}$ \\
\hline Multiple Sclerosis & $28(21.7 \%)$ \\
\hline CNS inflammatory-demyelinating diseases ${ }^{1}$ & $32(24.8 \%)$ \\
\hline CNS infections $^{2}$ & $9(7 \%)$ \\
\hline Tumours & \\
\hline Inflammatory PNS diseases $^{3}$ & $8(6.2 \%)$ \\
\hline CNS autoimmune/paraneoplastic neurological diseases $^{5}$ & $6(4.7 \%)$ \\
\hline Miscellaneous diagnoses & \\
\hline
\end{tabular}

${ }^{1}$ clinically isolated syndrome, radiologically isolated syndrome and neuromyelitis optica spectrum disorder; ${ }^{2}$ Borrelia burgdorferi, Herpes simplex virus, John Cunningham virus, treponema pallidum; ${ }^{3}$ tumours within/adjacent to the CNS: cerebral marginal zone lymphoma, cerebral glioma, dysembryoplastic neuroepithelial tumour, vertebral metastasis; hematologic tumours: chronic lymphocytic leukemia, Non-Hodgkin lymphoma, hepatic-pulmonary lymphoma; ${ }^{4}$ Guillain-Barrè syndrome, anti-MAG neuropathy, multifocal motor neuropathy, chronic inflammatory demyelinating polyneuropathy; ${ }^{5}$ neuro-systemic lupus erithematosus, Bickerstaff encephalitis, anti-phospholipid syndrome, cerebral vasculitis, anti- N-methyl D-aspartate receptor antibody encephalitis; ${ }^{6}$ idiopathic intracranial hypertension, spondilomyelopathy, cerebral ischaemia, vascular encephalopathy, amyotrophic lateral sclerosis, polyneuropathy, Alzheimer's disease, fronto-temporal dementia, neurosarcoidosis, Tolosa-Hunt syndrome, cerebral autosomal dominant arteriopathy with subcortical infarcts and leukoencephalopathy, Melkersson-Rosenthal syndrome, reversible cerebral vasoconstriction syndrome, HCVrelated multineuropathy, DYT1-positive dystonia, cerebral venous thrombosis, migraine, tension-type headache, syndrome of transient headache and neurologic deficits with cerebrospinal fluid lymphocytosis, spinal dural fistula, psychiatric conditions (catatonia, conversion disorder), post-traumatic epilepsy, perinatal paraparesis, transient global amnesia 
Table 2 Demographic and cerebrospinal fluid characteristics of patients with a single cerebrospinal fluid IgG band with multiple sclerosis, CNS inflammatory-demyelinating and miscellaneous diseases

\begin{tabular}{|c|c|c|c|c|}
\hline & $\begin{array}{c}\text { Multiple } \\
\text { sclerosis } \\
\quad(n=28)\end{array}$ & $\begin{array}{c}\text { CNS inflammatory- } \\
\text { demyelinating } \\
\text { diseases } \\
(n=32)\end{array}$ & $\begin{array}{c}\text { Miscellaneous } \\
\text { diseases } \\
(n=69)\end{array}$ & p-value \\
\hline Age (years) (mean $\pm \mathrm{SD})$ & $37.7 \pm 13$ & $37.2 \pm 15$ & $50.3 \pm 19$ & $* 0.003$ \\
\hline Sex (Male/Female) & $13 / 15$ & $10 / 22$ & $27 / 42$ & ns \\
\hline $\begin{array}{l}\text { CSF cell count (number/ } \mu \text { l), } \\
\text { median (IQR) }\end{array}$ & $3(1-7)$ & $1.5(1-3.5)$ & $1(1-2)$ & ns \\
\hline $\begin{array}{l}\text { CSF total protein }(\mathrm{g} / \mathrm{dl}) \text {, } \\
\text { median }(\mathrm{IQR})\end{array}$ & $47(27-59)$ & $37(33-49)$ & $37(30-48)$ & ns \\
\hline IgG index, median (IQR) & $0.53(0.5-0.65)$ & $0.56(0.5-0.6)$ & $0.58(0.5-0.66)$ & ns \\
\hline $\begin{array}{l}\text { CSF/serum albumin, } \\
\text { median (IQR) }\end{array}$ & $5(3.1-6.7)$ & $4.7(3.6-6.3)$ & $4.6(3.7-6.3)$ & ns \\
\hline $\begin{array}{l}\text { Additional mirror pattern } \\
\text { (yes/no) }\end{array}$ & $2 / 26$ & $3 / 29$ & $17 / 49$ & $\infty 0.04$ \\
\hline
\end{tabular}

$\mathrm{SD}=$ standard deviation; $\mathrm{IQR}=$ interquartile range

*post-hoc tests: MS versus CNS inflammatory-demyelinating diseases: $\mathrm{p}=0.45$; CNS inflammatory-demyelinating diseases versus miscellaneous diseases: $\mathrm{p}=0.0003$; MS versus miscellaneous diseases: $\mathrm{p}=0.001$

$\infty$ post-hoc tests: MS versus CNS inflammatory-demyelinating diseases: $\mathrm{p}=0.6$; CNS inflammatory-demyelinating diseases versus miscellaneous diseases: $p=0.048$; MS versus miscellaneous diseases: $p=0.032$ 
Table 3. Studies exploring the frequency and diagnostic meaning of single cerebrospinal fluid-restricted IgG bands

\begin{tabular}{|c|c|c|c|c|c|c|c|}
\hline Authors & N/total (\%) & $\begin{array}{c}\text { MS } \\
\text { N (\%) }\end{array}$ & $\begin{array}{c}\text { CNS } \\
\text { inflammatory } \\
\text { / } \\
\text { autoimmune/ } \\
\text { paraneoplasti } \\
\text { c disease } \\
\mathrm{N}(\%)\end{array}$ & $\begin{array}{c}\text { PNS } \\
\text { inflammato } \\
\text { ry disease } \\
\text { N }(\%)\end{array}$ & $\begin{array}{c}\text { CNS } \\
\text { infection } \\
\mathrm{N}(\%)\end{array}$ & $\begin{array}{c}\text { CNS tumor } \\
\text { N }(\%)\end{array}$ & $\begin{array}{c}\text { Seemingly } \\
\text { unrelated } \\
\text { disease } \\
\text { N (\%) }\end{array}$ \\
\hline $\begin{array}{c}\text { McCombe et al } \\
{[8]}\end{array}$ & $\begin{array}{l}3 / 1490 \\
(0.2 \%)\end{array}$ & - & $1(33)$ & $1(33)$ & - & $\begin{array}{c}1(33) \\
\text { (lymphoma) }\end{array}$ & - \\
\hline Ben-Hur et al [4] & $\begin{array}{c}33 / 6000 \\
(0.6 \%) \\
\text { (20 with } \\
\text { diagnosis) }\end{array}$ & $7(35)$ & $9(45)$ & $\begin{array}{c}3(15) \\
\left(\begin{array}{c}1 \text { had MS } \\
\text { too })\end{array}\right.\end{array}$ & - & - & $2(10)^{1}$ \\
\hline $\begin{array}{c}\text { Franciotta et al } \\
\text { [7] }\end{array}$ & $\begin{array}{l}61 / 3273 \\
(1.9 \%)\end{array}$ & $27(44)$ & $8(13)$ & $1(2)$ & $16(26)$ & - & $9(15)^{2}$ \\
\hline Davies et al [5] & $\begin{array}{c}31 \\
\text { (27 with } \\
\text { diagnosis) }\end{array}$ & $3(11)$ & $7(26)$ & - & $4(15)$ & $\begin{array}{c}1(4) \\
\text { (lymphoma) }\end{array}$ & $12(44)^{3}$ \\
\hline
\end{tabular}

$\mathrm{N}=$ number; ${ }^{1}$ torsion dystonia, superficial hemosiderosis of the CNS; ${ }^{2}$ polyneuropathy ( $\mathrm{n}=5$ ), Alzheimer's disease $(n=2)$, retrobulbar optic neuropathy $(n=2) ;{ }^{3}$ axonal neuropathy $(n=2)$, vascular disease $(n=2)$, other/no diagnosis $(n=8)$ : orthostatic tremor, migraine+/-, oscillopsia, intermittent sensory symptoms, cerebral venous infarction, progressive cerebellar syndrome, chronic myalgia 